\section{Spinal abscess and mitral valve endocarditis secondary to asymptomatic fusobacterium-induced dental abscess}

\section{S I Goolamali M T Carulli U M Davies}

J R Soc Med 2006;99:368-369

Fusobacterium nucleatum is an anaerobic bacillus of oropharyngeal origin. We describe a case of two manifestations of fusobacterium-induced sepsis originating from an asymptomatic dental root abscess.

\section{CASE HISTORY}

A 63-year-old Caucasian man presented to the accident and emergency department with a 2-week history of night sweats, anorexia, $6 \mathrm{~kg}$ weight loss and lumbo-sacral back pain radiating to the right buttock and thigh. In addition, he had been intermittently confused and drowsy over the previous 48 hours. Other than gout and osteoarthritis there was no significant past medical history. On examination, he was apyrexial but tachycardic, with normal cardiac sounds and no peripheral stigmata of infective endocarditis. There was no focal spinal tenderness or nuchal rigidity and neurological examination was normal apart from significantly diminished straight leg raise on the right. Laboratory tests showed raised inflammatory markers (C-reactive protein [CRP] $402 \mathrm{mg} / \mathrm{L}$, normal range $[\mathrm{NR}]<10$; erythrocyte sedimentation rate [ESR] $84 \mathrm{~mm}$ in first hour), leucocytosis, (total white cell count [WCC] 27.5 with neutrophils $23.1 \times 10^{9} / \mathrm{L}$ ) and deranged liver function (alkaline phosphatase $282 \mathrm{IU} / \mathrm{L}, \mathrm{NR}<125$; AST $53 \mathrm{IU} / \mathrm{L}$, NR < 40; LDH 225 IU/L, NR <180). Serial blood cultures, myeloma screen, tumour markers, X-ray of the chest and lumbo-sacral spine, abdominal ultrasound and electrocardiogram were negative or normal. Magnetic resonance imaging of the lumbar spine showed discitis of L4/L5. This disc was subsequently aspirated under computed tomography guidance prior to commencement of empirical antibiotic therapy with intravenous flucloxacillin and oral Fucidin (sodium fusidate).

Department of Rheumatology, East Surrey Hospital, Redhill, Surrey RH1 5RH, UK Correspondence to: Dr S I Goolamali, Department of Dermatology, Chelsea and Westminster Hospital, London SW10 9NH, UK
Forty-eight hours later the patient became acutely confused with signs of acute urinary retention and bilateral lower limb hypertonia and hyperreflexia. Plantar responses were flexor and muscle power normal; sensation was difficult to assess. Repeat blood tests demonstrated a rise in neutrophil count to $32.9 \times 10^{9} / \mathrm{L}$ and acute renal failure with urea $24.1 \mathrm{mmol} / \mathrm{L}$ and creatinine $409 \mu \mathrm{mol} / \mathrm{L}$. Lumbar spine magnetic resonance imaging was repeated and now showed a paravertebral abscess at the L4/L5 level (Figure 1). The patient underwent urgent right L5 laminectomy with exploration of the epidural space at L4/L5 and L5/S1. Fusobacterium nucleatum was isolated from both the pus drained at operation and the original computerized tomograph-guided aspirate. Antibiotic therapy was changed to metronidazole and benzylpenicillin, to which the organism was sensitive. Urea and creatinine normalized with inotropic support and diuretics.

Following his initial recovery the patient deteriorated acutely with pyrexia and respiratory failure requiring ventilatory support. Cardiac sounds were normal, serial blood cultures remained negative and computerized tomograph of the brain was normal. Echocardiography revealed vegetations on the mitral valve indicative of infective endocarditis and gentamicin therapy was added.

After extubation, the patient's teeth were routinely examined and a previously undetected asymptomatic dental root abscess was found, thought to be the most likely source of the $F$. nucleatum. Further recovery was uneventful and he underwent elective dental treatment of the abscess. Follow up magnetic resonance imaging of the lumbar-spine two months later was normal.

\section{COMMENT}

Fusobacterium spp is one of only four genera of anaerobic Gram-negative bacilli which are thought to be responsible for human infection. It produces a virulence factor liposaccharide endotoxin, which contributes to abscess formation. ${ }^{1}$ Fusobacterium nucleatum comprises part of the normal oropharyngeal bacterial flora and is a recognized pathogen in odontogenic infection. Anaerobic Gramnegative bacilli causing odontogenic orofacial infections are associated with more severe illness than other bacterial species, with $F$. nucleatum highlighted as a particularly potent pathogen. ${ }^{2}$ Our patient's dental root abscess, almost certainly the likely source of the bacillus, was picked up incidentally and he was entirely asymptomatic. Fusobacterium spp particularly Fusobacterium necrophorum is a recognized cause of postanginal sepsis or Lemierre's syndrome, characterized by bacteraemia with metastatic abscesses following an acute sore throat. ${ }^{3}$ It has recently been postulated that $F$. necrophorum is a potent pathogen and often 


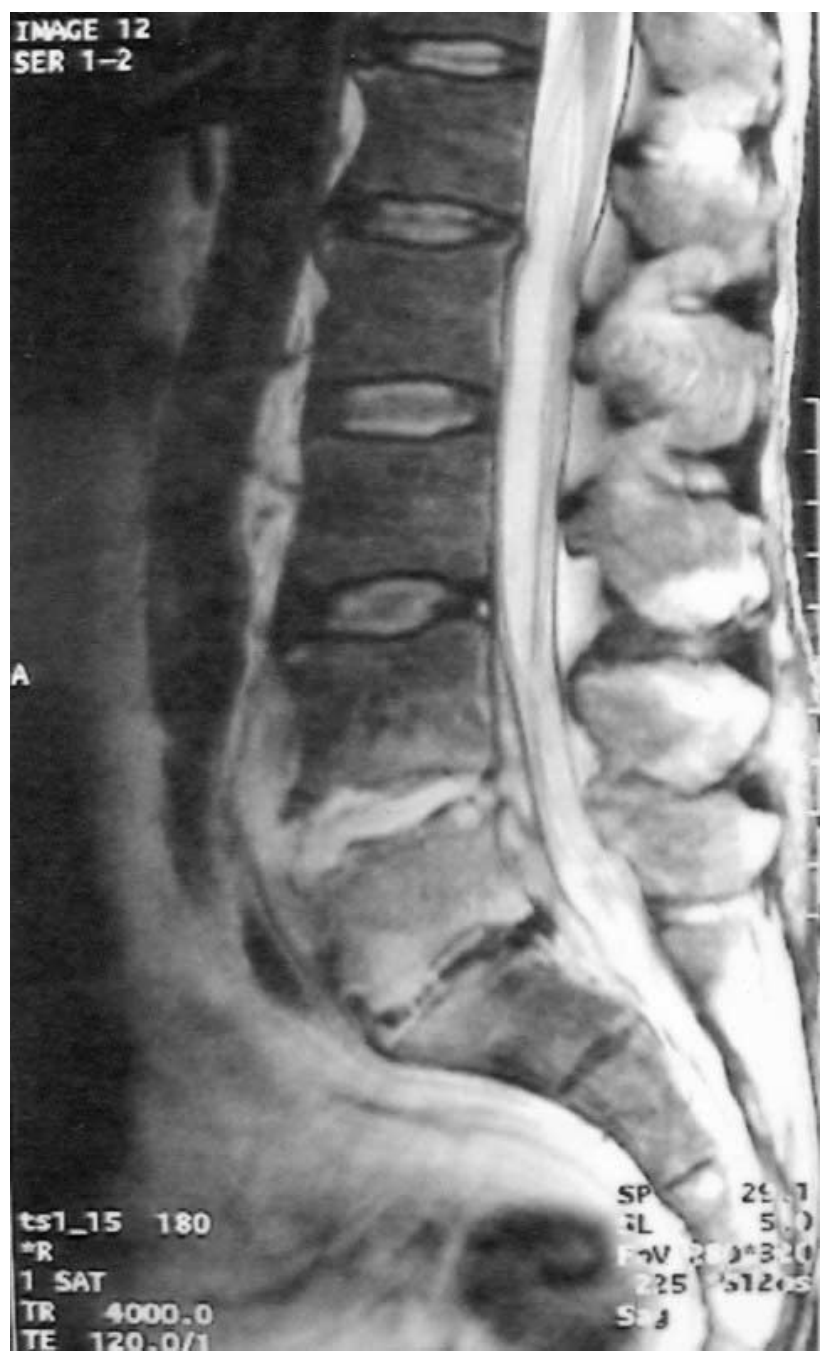

Figure 1 Lumbar spine magnetic resonance imaging demonstrating epidural abscess at the L4/L5 disc level

induces the 'simple' sore throat in the community without co-existing sepsis. ${ }^{4}$

Anaerobic Gram-negative bacilli may frequently cause cerebral abscesses and, to a lesser extent, subdural empyemas and cranial epidural abscesses. Spinal epidural abscess is not commonly associated with these organisms: ${ }^{5}$ though acute and chronic cases can arise by haematogenous spread, either by direct seeding of the epidural space or by producing vertebral osteomyelitis (discitis) with subsequent epidural space extension. In our case, the source of infection was dental and, although the patient was on established penicillin therapy without preceding penetrating trauma or evidence of contiguous site infection, haematogenous spread was probably the mode of transmission. In the absence of any other focal infection, it is reasonable to assume that a transient bacteraemia from the dental or epidural abscess also caused the endocarditis.

Infective endocarditis caused by anaerobic bacteria is uncommon with reported incidences varying from $1 \%-16 \%$ of all cases. ${ }^{1}$ Bacteroides fragilis and streptococci are the most frequent causes. ${ }^{6}$ Endocarditis caused by F. nucleatum is extremely rare with only a few cases reported in the literature. ${ }^{7}$

Our patient developed focal pain for 2 weeks prior to the rapid development of radicular signs, cord compression and dissemination which forms the classic clinical presentation of an epidural abscess arising from vertebral osteomyelitis. Acute metastatic epidural space infection produces rapid progression with systemic signs of infection and localized pain predominating. ${ }^{1}$ Mortality from epidural abscess is high and early diagnosis is essential. Fusobacterium is an unusual cause for both an epidural abscess and endocarditis and emphasizes the importance of considering a spinal abscess and careful cardiac monitoring of patients complaining of focal back pain with concomitant evidence of sepsis.

Competing interests None declared.

\section{REFERENCES}

1 Mandell GL, Bennett JE, Dolin R eds. Principles and Practice of Infectious Diseases, 5th edn. New York:Churchill Livingstone, 2000;237: 2561-8

2 Heindahl A, von Konow L, Satoh T, et al. Clinical appearance of orofacial infections of odontogenic origin in relation to microbiological findings. J Clin Microbiol 1985;22:299-302

3 Shah SA, Ghani R. Lemierre's syndrome: a forgotten complication of oropharyngeal infection. J. Ayub Med Coll Abbottabad 2005;17:30-3

4 Aliyu SH, Marriott RK, Curran MD et al. Real-time PCR investigation into the importance of Fusobacterium necrophorum as a cause of acute pharyngitis in general practice. J Med Microbiol 2004;53:1029-35

5 Swartz MN. Central nervous system infections. In: Finegold SM, George WL, eds. Anaerobic Infections in Humans. New York: Academic, 1989:155-212

6 Felner JM, Dowell VR Jr. Anaerobic bacterial endocarditis. N Eng J Med 1970;282:1188-92

7 Shammas NW, Murphy GW, Eichelberger J, et al. Infective endocarditis due to Fusobacterium nucleatum: case report and review of the literature. Clin Cardiol 1993;16:72-5 


\section{Trigger thumb in a fish-owner}

\section{A Abood N Kang}

J R Soc Med 2006;99:370

Mycobacterium marinum is an uncommon but well documented infection in man. It has the ability to hide behind many guises, which can make diagnosis difficult.

\section{CASE HISTORY}

A woman aged 74 was referred because of stiffness and triggering of her right thumb (dominant hand). On examination there was no evidence of inflammation; triggering was evident in the absence of palpable Notta's nodule. Radiographs of the thumb and hand showed nothing of note. Treatment options were discussed, including injection of steroids into the affected thumb to relieve triggering, but the patient declined at that stage and made an appointment to return two weeks later. By the time of her second visit she had developed a well circumscribed, nodular lesion over the dorsum of the first web space of her right hand and similar lesions tracking up her arm (Figure 1a). On detailed inquiry she then revealed that four weeks before her initial presentation she had sustained a minor laceration to the thumb after striking a metal object; moreover, she kept tropical fish and had continued to handle the aquarium without gloves.

Blood tests including white cell count and C-reactive protein were within normal limits. A single lesion was excised and sent for histological and microbiological examination. Culture grew Mycobacterium marinum and, on the recommendation of the microbiologist, the patient began treatment with clarithromycin, rifampicin and ethambutol. Four weeks into treatment the triggering of her thumb resolved.

\section{COMMENT}

M. marinum is a Runyon group I photochromogenic mycobacterium that causes tuberculosis in fish. ${ }^{1}$ It grows at $30-32^{\circ} \mathrm{C}$, about five degrees less than typical mycobacteria, and this may explain why it is frequently confined to the superficial structures in the limbs. ${ }^{2}$ Infection in man typically begins with a single, papulonodular granulomatous lesion (Figure $1 b$ ) but can progress to a sporotrichoid form in a single limb (as in our patient). ${ }^{3}$ The

Department of Plastic, Reconstructive \& Burns Surgery, Mount Vernon Hospital, Northwood HA6 2RN, UK

Correspondence to: Mr A Abood

E-mail: ahidabood@yahoo.co.uk (a)

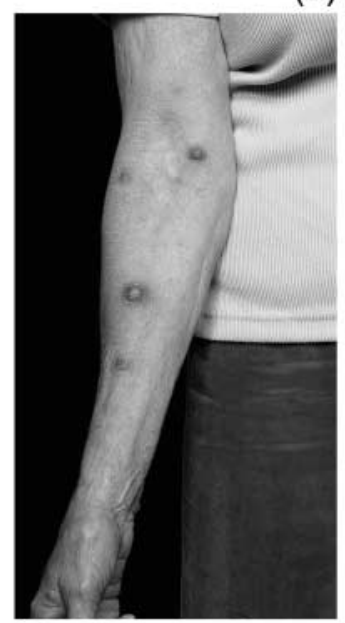

(b)

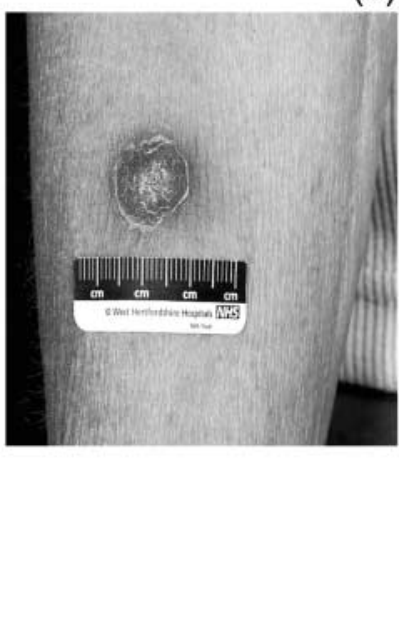

Figure 1 (a) Proximal migration of lesions; (b) single lesion

main differential diagnosis is indeed sporotrichosis, which tends to affect gardeners. The cutaneous expression of M. marinum infection is known as fish tank or swimming pool granuloma. In the initial stages of infection, the resemblance of symptoms to inflammatory joint disease has prompted administration of intralesional steroids, with consequent exacerbation. ${ }^{1,2,4,7}$

Contrary to the opinion expressed in certain reports, ${ }^{1,5}$ $M$. marinum does not restrict itself to superficial structures. It can present with tenosynovitis, bursitis, gout, rheumatoid arthritis, and even destructive polyarthritis. ${ }^{3,4,6,7}$ The unwary may treat these with local steroids and make matters worse. When the diagnosis has been made, the usual treatment is excision and chemotherapy. Occasionally, as in our case, antimicrobial treatment alone has sufficed. This case adds to the two previously reported cases of trigger finger caused by $M$. marinum.

Competing interests None declared.

\section{REFERENCES}

1 Lim YW, Chia J, Looi KP. A case report of Mycobacterium marinum infection of the hand. Singapore Med J 2000;41:221-3

2 Williams CS, Riordan DC. Mycobacterium marinum infections of the hand. J Bone Joint Surg 1973;55A:1042

3 Chow SP, Stroebel AB, Lau JHK, Collins RJ. Mycobacterium marinum infection of the hand involving deep structures. J Hand Surg 1983;8:568-73

4 Wendt JR, Lamm RC, Altman DI, Cruz HG, Achauer BM. An unusually aggressive Mycobacterium marinum hand infection. J Hand Surg 1986;11:753-5

5 Gray SF, Smith RS, Reynolds NJ, Williams EW. Fish tank granuloma. BMJ 1990;300:1069-70

6 Winter FE, Runyon EH. Prepatellar bursitis caused by Mycobacterium marinum. Case report, classification and review of the literature. $J$ Bone Joint Surg 1965;47A:375-9

7 Klinberg JR, Grimley PM, Seegller JE. Destructive polyarthritis due to a photochromogene mycobacterium. N Engl J Med 1965;272:190 


\section{Syphilis presenting as acute multifocal retino-choroiditis}

\author{
Shery Thomas ${ }^{1} \quad$ Martin Wiselka² Jyoti Dhar ${ }^{3}$ \\ Kim Bibby ${ }^{1}$
}

J R Soc Med 2006;99:371-372

We describe a patient who presented with an unusual bilateral migrating luetic chorioretinitis.

\section{CASE HISTORY}

A 44-year-old celibate Caucasian man presented with a 1day history of decreased left vision. There was no significant past history. Visual acuities were $6 / 6$ in the right eye and $6 / 36$ in the left. There were cells $(+)$ in the left vitreous and an area of chorio-retinitis below the left macula. A fluorescein angiogram showed associated extensive leakage. Routine blood tests, including full blood count and Creactive proteins, were normal. The chest X-ray was unremarkable. His serum rheumatoid factor was 260 IU/ $\mathrm{mL}(N=<20)$; autoantibody screening was negative; and the serum angiotensin converting enzyme was raised at 62 $(N=8-52)$. A diagnosis of idiopathic inflammatory chorioretinitis was made.

On commencing oral prednisolone $60 \mathrm{mg} /$ day the chorioretinitis subsided and visual acuity improved to 6/ 6 . The systemic steroids were reduced and 2 weeks later vision in the right eye deteriorated to 6/24. Examination showed mild vitritis and the fluorescein angiogram indicated late leakage at the right posterior pole. Intravenous methylprednisolone $1 \mathrm{~g} /$ day was commenced for 3 days, and the visual acuity in the right eye improved to $6 / 9$. He was maintained on oral steroids $(20 \mathrm{mg} /$ day $)$ and cyclosporin (150 mg/day); but further visual deterioration occurred over the next 10 days to hand movements in both eyes. He exhibited a migrating multifocal retinitochoroiditis with whitish placoid retinal lesions (Figure 1 [top] and Figure 2). The peripheral phlebitis and mild disc oedema were noted bilaterally.

Serology excluded toxoplamosis, Lyme disease and Weil's disease. A polymerase chain reaction was done on the cerebrospinal fluid to exclude herpes simplex, varicella zoster and cytomegalovirus. An echoencephalogram was normal.

Departments of ${ }^{1}$ Ophthalmology, ${ }^{2}$ Infectious Diseases, ${ }^{3} \mathrm{GU}$ Medicine, Leicester Royal Infirmary, Leicester LE1 5WW, UK

Correspondence to: Miss Kim Bibby

E-mail: rosinaellen@aol.com

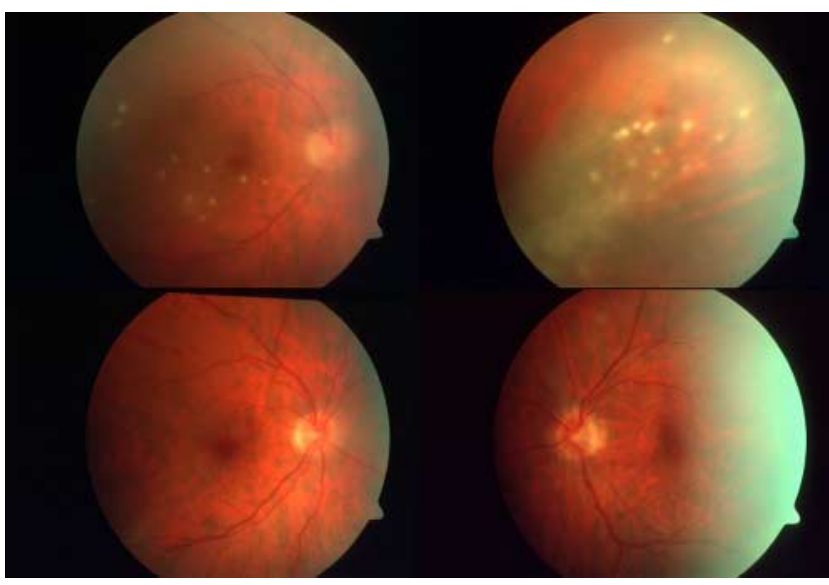

Figure 1 A fundus picture (both eyes). Top: showing multifocal placoid retino-choroiditis. Bottom: 4 weeks after commencing penicillin showing pigmentary changes at the maculae [in colour online]

Three months after initial presentation he developed a generalized maculo-papular rash with involvement of the palms, soles, lymphadenopathy and oral ulcerations. There was no evidence of genital ulceration. Although not deemed at risk of sexually transmitted infection, syphilis serology was also undertaken. Treponemal IgG and triethylene thiophosphoramide were positive with a VDRL of 1:64. Lumbar puncture found cerebrospinal fluid lymphocytosis, raised proteins and a positive Treponemal IgG.

He was admitted for a 17-day course of intravenous benzyl penicillin for the treatment of secondary
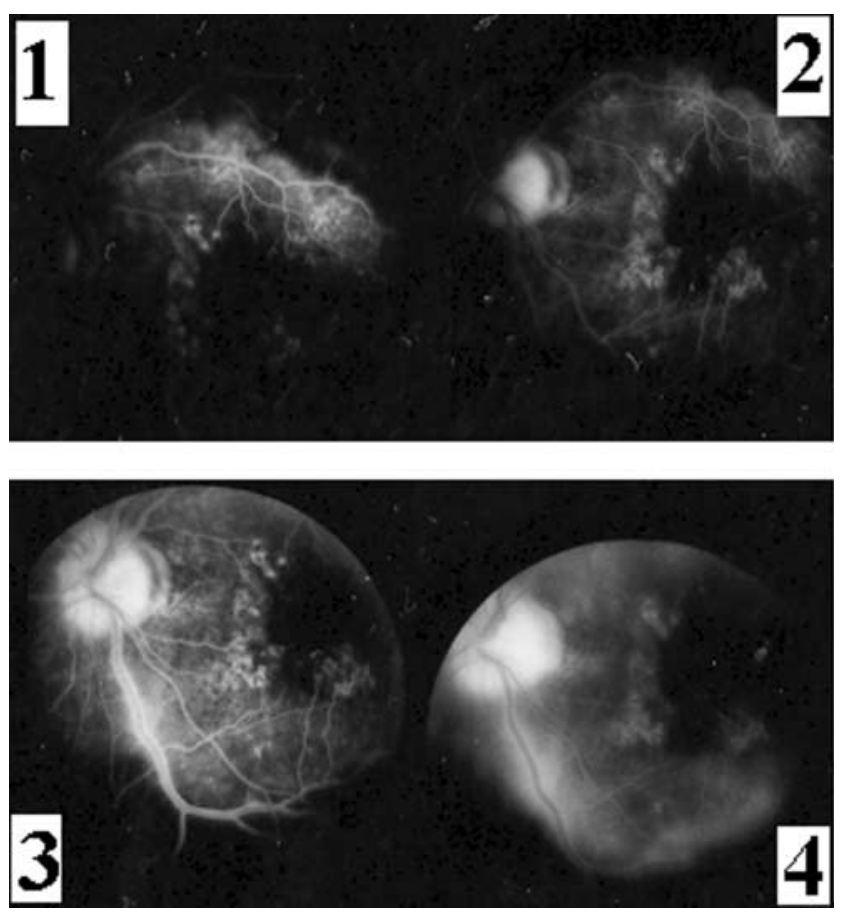

Figure 2 A fluorescein angiogram of the left eye showing leakage. Early and late phases are indicated by numbers: 1 being the earliest phase and 4 being the last 
neurosyphilis. A full sexual history was taken at this stage, and it was found that 3 months earlier he had been sexually assaulted by two men, which had involved unprotected receptive oral and anal intercourse. There had been no other sexual encounters with men or women for several years prior. Genitourinary screen and HIV testing were negative. (The UK national guidelines on the management of syphilis are available at [http://www.bashh.org].

Within a week his retinal oedema subsided. Within 3 weeks his retinal lesions had disappeared. His visual acuity improved to $6 / 9$ bilaterally. At last review mild residual pigmentary changes at both fovea persist (Figure 1 [bottom]). Follow-up syphilis serology at 3, 6 and 12 months showed a gradual drop in VDRL titres becoming serofast at a level of $1: 8$.

\section{DISCUSSION}

Our patient was a Caucasian man, who had been celibate for several years, presenting with acute multifocal migratory retino-choroiditis secondary to syphilis.

Syphilis had been recognized as a common cause of intraocular inflammation during the early half of the 20th century, but the occurrence declined dramatically with the advent of penicillin. The past decade has seen the reemergence of infectious syphilis in the UK. ${ }^{1-3}$ Primary syphilis is characterized by a chancre and lymphadenopathy; secondary syphilis is a systemic illness with a characteristic maculopapular rash and a wide range of systemic involvement including ocular disease. The most common ocular manifestation is anterior uveitis, though posterior, intermediate and panuveitis have also been described. Optic neuritis, scleritis and interstitial keratitis are less common. ${ }^{4}$
Diagnosis of syphilis was delayed in our patient for several reasons. Chorioretinitis as the initial presentation though described is rare. ${ }^{5}$ The clinical course of the disease was no doubt confounded by the use of immunosuppressants, and the deemed irrelevance of sexual history taking to the presenting complaint.

Although the majority of recent syphilis cases have been diagnosed at genitourinary clinics in the UK, ${ }^{2}$ it seems likely that the cases of secondary syphilis, especially if associated with less of the usual symptoms, may present to other specialities including eye departments. ${ }^{6}$

Early screening for syphilis should be routine for any unusual cause of uveitis, however improbable the diagnosis may seem. A missed diagnosis can lead to devastating ocular and systemic consequences.

Competing interests None declared.

\section{REFERENCES}

1 Brown AE, Sadler K E, Tomkins S E, et al. Recent trends in HIV and other STIs in the United Kingdom: data to the end of 2002. Sex Transm Infect 2004;80:159-66

2 Ashton M, Sopwith W, Clark P, McKelvey D, Lighton L, Mandal D. An outbreak no longer: factors contributing to the return of syphilis in Greater Manchester. Sex Transm Infect 2003;79:291-3

3 Fenton KA, Nicoll A, Kinghorn G. Resurgence of syphilis in England: time for more radical and nationally co-ordinated approaches. Sex Transm Infect 2001;77:309-10

4 Ormerod LD, Pulkin JE, Sobel JD. Syphilitic posterior uveitis: correlative findings and significance. Clin Infect Dis 2001;32:1661-73

5 Zamani M, Garfinkel RA. Corticosteroid-induced modulation of acute syphilitic posterior placoid chorioretinitis. Am J Ophthalmol 2003;135: $891-4$

6 Poitevin M, Collart P, Bolgert M. Syphilis in 1986. J Clin Neuro Ophthalmol 1987;7:11-19 